\title{
Maladies des plantes dues à Rhizoctonia solani (Kühn) : stratégie et techniques d'étude - résultats
}

\author{
P. Camporota \\ INRA, Station de recherches sur la flore pathogène dans le sol, 17, rue Sully, 21034 Dijon Cedex, France
}

(reçu le 6-6-1988, accepté le 6-1-1989)

Résumé - Les techniques présentées permettent la détermination fine des souches de $R$. solani et l'appréciation de l'influence exercée par le sol sur le saprophytisme et le parasitisme du champignon. Les résultats obtenus et l'intérêt d'utiliser ces techniques pour la recherche de voies de lutte sont discutés.

potentiel infectieux - capacité saprophytique - fongicides - lutte - Trichoderma spp. - Thanatephorus cucumeris

Summary - Plant diseases induced by Rhizoctonia solani (Kühn) : strategy and methods of study. Results. The proposed techniques allow an accurate determination of $\mathrm{R}$. solani strains and assessment of soil influence on the parasitic and saprophytic behaviour of the fungus. Results obtained and utilization of these techniques to improve control are discussed.

soil infectivity - competitive saprothytic ability - control - Trichoderma sp. - Thanatephorus cucumeris

\section{Introduction}

Au cours des dernières années, les interventions de la profession agricole demandant de résoudre des problèmes posés par Rhizoctonia solani ont notablement augmenté. Que ce soit en cultures maraîchères (laitue, chicorée, radis), en floriculture (impatiens, giroflée, chrysanthème) ou en grandes cultures (pomme de terre, maïs, betterave sucrière), ce champignon pathogène du sol cause des pertes dont l'importance croissante justifie les recherches qui lui sont consacrées.

Au cours des travaux menés dans notre laboratoire, nous avons établi un plan d'étude de ces maladies utilisant les techniques que nous avons mises au point; ce plan permet de réaliser les étapes classiques en pathologie végétale : connaissance du parasite, épidémiologie, lutte.

Dans cet article, nous exposons la réalisation de ces différentes étapes, les techniques utilisées avec leurs améliorations, enfin des résultats acquis.

\section{l. Connaissance de l'agent pathogène}

1) Isolement

\section{Sur le végétal}

Les symptômes sont assez faciles à reconnaître et, de même, l'isolement sur milieu gélosé (PDA ou malt, acidifié ou additionné d'antibiotiques) est relativement aisé.

\section{Dans le sol}

Des pièges, constitués par des rondelles de papier filtre imprégnées par un milieu sélectif, sont mis en contact pendant 5 jours avec le sol portant le végétal attaqué; ils sont ensuite retirés et déposés sur un milieu gélosé sélectif $\left(\mathrm{K}_{2} \mathrm{H} \mathrm{PO}_{4}: 1 \mathrm{~g} ; \mathrm{Mg} \mathrm{SO}_{4}: 0,5 \mathrm{~g}\right.$; $\mathrm{KCl}: 0,5 \mathrm{~g} ; \mathrm{Fe} \mathrm{SO}_{4}: 0,01 \mathrm{~g} ; \mathrm{Na} \mathrm{NO}_{2}: 0,2 \mathrm{~g}$; acide tannique: $0,12 \mathrm{~g}$; propamocarbe (Schering) : $0,8 \mathrm{~g}$; sulfate de streptomycine: $0,1 \mathrm{~g}$; chlortétracycline: $0,05 \mathrm{~g}$; étridiazole (Seppic): 0,005 g; $\mathrm{Cu} \mathrm{SO} 4: 0,0013 \mathrm{~g}$; bénomyl (Dupont): $0,0013 \mathrm{~g}$; gélose $15 \mathrm{~g} ; \mathrm{H}_{2} \mathrm{O}$ qsp: $1000 \mathrm{ml}$; stérilisation $30 \mathrm{~min} / 110^{\circ} \mathrm{C}$ ) pour mettre le champignon en évidence (Camporota, 1981).

En confrontant chaque piège à une quantité égale de sol, il est possible de déterminer le nombre le plus probable de propagules par gramme (Ricci, 1972). 


\section{2) Identification des souches}

\section{a) Appartenance à l'espèce R. solani}

Parmeter et Whitney (1970) ont défini un certain nombre de critères morphologiques pour déterminer R. solani. Mais cette forme végétative stérile est commune à 2 basidiomycètes: Thanatephorus cucumeris (Frank-Donk) et Ceratobasidium sp. (Rodger). Les souches rattachées à $T$. cucumeris correspondent à l'espèce $R$. solani et possèdent plus de deux noyaux par article mycélien; celles rattachées à Ceratobasidium $\mathrm{sp}$. sont toujours binucléées (Camporota et al., 1984).

Une technique simple permet de dénombrer les noyaux et de départager les souches des 2 genres: sur la frange de cultures réalisées sur milieu malt 1,5\% en couche mince, on dépose une goutte de bleu d'aniline à $1 \%$ en solution alcoolique à $2 \%$. Après couverture par une lamelle, on dénombre les noyaux au microscope $(G \times 40)$ au bout de 2 à $4 \mathrm{~h}$ de coloration à la température du laboratoire sous éclairage (Fig. 1).

\section{b) Appartenance à un groupe d'anastomose}

Les souches de $R$. solani peuvent être classées dans des groupes biologiquement distincts en les confrontant, sur eau gélosée $(1,5 \%)$ en couche mince, à des souches de références et en observant la présence ou l'absence d'anastomoses entre leurs hyphes (Fig. 2 et 3) (Parmeter et al. 1969). En Europe, existent 4 principaux groupes d'anastomose : AG1 à AG4 (AG : Anastomosis Grouping), possédant une spécificité parasitaire plus ou moins marquée : très forte pour AG3 (souches inféodées aux Solanées et surtout à la pomme de terre), faible à moyenne pour AG2 (souches généralement agressives sur Crucifères mais aussi sur le maïs et la betterave sucrière), presque nulle pour AG1 et AG4 dont les souches sont polyphages (Camporota et al., 1984).

\section{3) Agressivité sur un hôte végétal}

Elle est appréciée en confrontant un inoculum identique pour chaque souche analysée (un explant calibré prélevé dans une culture sur milieu gélosé) à une population de plantules sensibles, dans des conditions d'environnement standardisées et optimales pour l'agressivité du parasite et la sensibilité de la plante. Trois espèces végétales ont été choisies: Vigna radiata (Wilczek) pour AG1 - AG4, Raphanus sativus pour AG2, germes de Solanum tuberosum pour AG3. Les

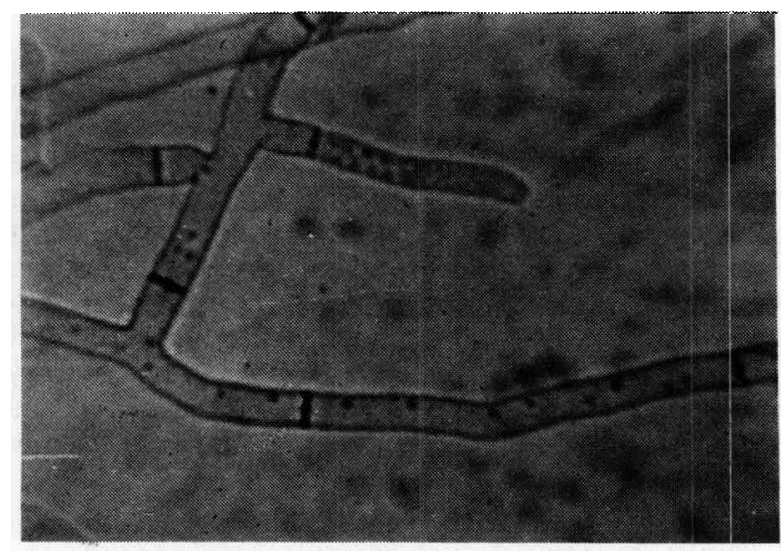

Fig. 1. Hyphes de $R$. solani multinuclées.

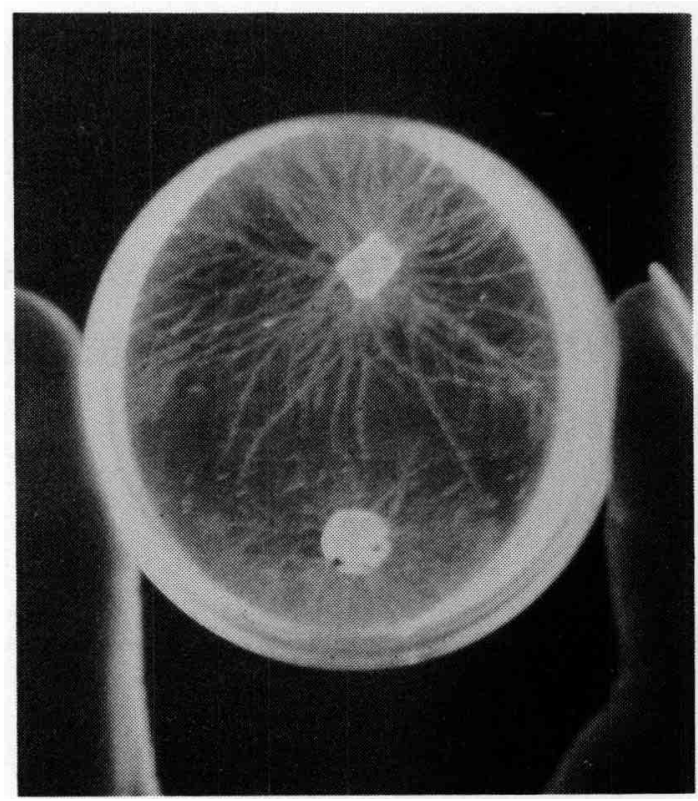

Fig. 2. Détermination du groupe d'anastomose: la souche de référence est située au bas.

résultats sont exprimés par un indice de maladie variant de 0 à 100 (Camporota, 1982; Aouchiche, 1982).

L'utilisation de ces différentes techniques permet d'obtenir 3 niveaux de renseignements concernant :

- la relation entre les souches isolées sur le végétal et celles présentes dans le sol qui le porte;

- la composition de la population et la proportion de souches agressives, pour un sol naturellement infesté par R. solani;

- le choix rapide des souches qui serviront pour les réinoculations, les études épidémiologiques et les essais de lutte.

\section{Epidémiologie - influence du sol sur le parasite}

\section{1) Principes d'étude}

Les recherches sur l'épidémiologie d'un champignon phytopathogène du sol consistent à essayer de connaître l'influence exercée par le milieu sol sur l'inoculum du parasite qu'il contient.

Une situation biologique intéressant la pathologie du sol, peut schématiquement être représentée comme un écosystème dont le fonctionnement dépend des interactions de quatre paramètres : la plante, le sol qui la porte, l'inoculum pathogène et les composantes climatiques. La résultante de ces interactions est mise en évidence par l'intensité de la maladie sur la plante. Pour connaître le poids du paramètre sol dans le fonctionnement de l'écosystème, il est logique de penser, qu'en maîtrisant convenablement les trois paramètres : inoculum, plante et les conditions climatiques, la gravité de la maladie sur la plante sera directement modulée par l'influence du sol sur l'inoculum du parasite.

Ce principe a été utilisé pour mettre au point des essais biologiques servant à mesurer le Potentiel 


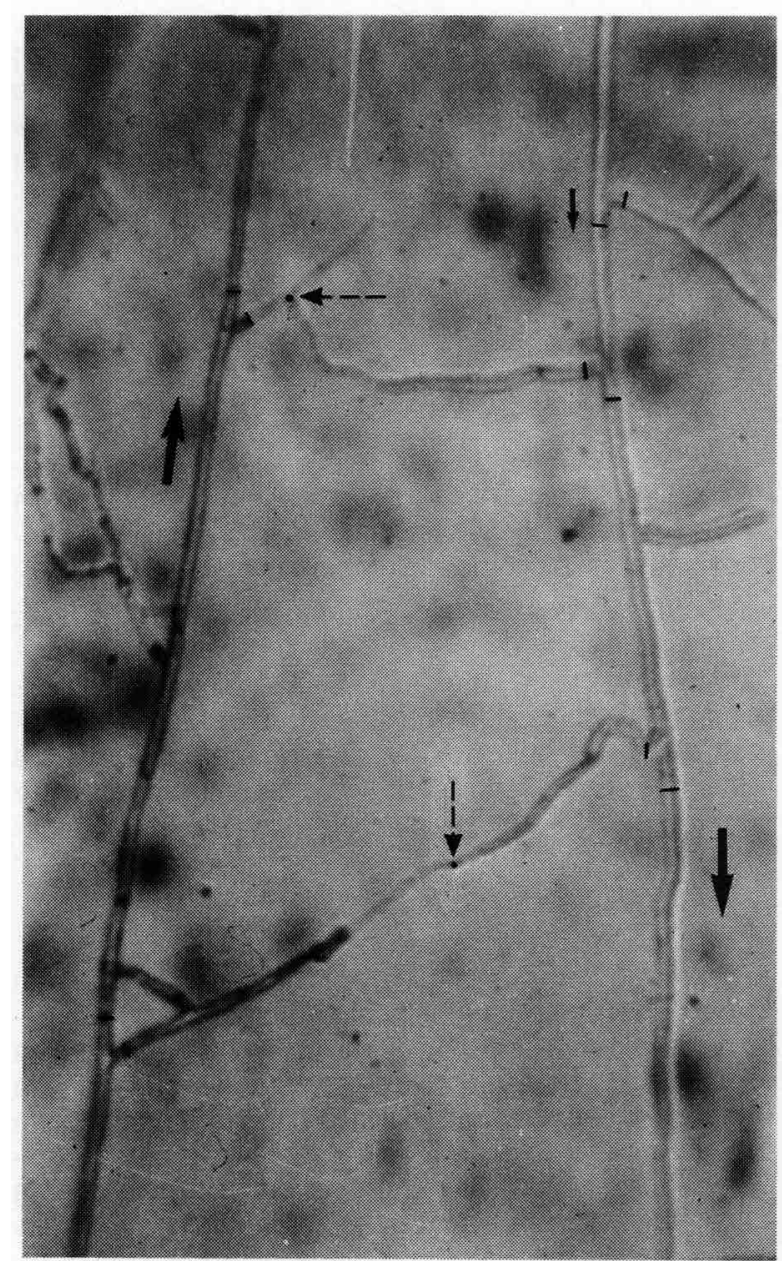

Fig. 3. Exemple d'anastomose: les flèches montrent le sens de croissance des hyphes qui s'anastomosent en 2 endroits (na) )

Infectieux $(\mathrm{PI})$ de sols infestés par l'inoculum d'une souche de $R$. solani dont on connaît l'appartenance à un groupe d'anastomose et l'agressivité : les conditions d'environnement (intensité lumineuse, humidité, température) ont été expérimentalement standardisées afin d'obtenir régulièrement l'attaque la plus élevée de la plantule sensible, compte tenu de la nature physique et biologique du sol. La mesure du PI est obtenue en 13 jours et les résultats sont exprimés en Unités de Potentiel Infectieux 50/g (UPI 50/g) (Camporota, 1982; Aouchiche, 1982).

L'inoculum d'une souche de $R$. solani possède deux caractéristiques biologiques: son agressivité et sa Capacité Saprophytique (CS). La mesure du PI d'un sol étant globale, elle ne permet pas de faire la part de chacune de ces caractéristiques. Or il est important de pouvoir apprécier la CS, en général élevée pour $R$. solani, étant donné qu'il a été démontré l'existence d'une liaison étroite entre le parasitisme et le saprophytisme pour ce champignon (Sneh et al., 1966; Papavizas, 1970; Geypens, 1974). Ceci est réalisé en remplaçant, dans l'écosystème décrit plus haut, la plante par un piège et en standardisant les conditions d'environnement : il est ainsi possible de mesurer l'influence exercée par le sol sur la CS d'une souche de $R$. solani. La mesure de capacité saprophytique est faite en 6 jours et les résultats sont exprimés en Unités de Colonisation 50/g (UC 50/g) (Camporota, 1981).

\section{2) Réceptivité du sol}

La réceptivité d'un sol à $R$. solani est appréciée en mesurant les variations de son PI au cours du temps. La première étape de l'étude consiste à produire, de façon standard, un inoculum à partir d'une souche de $R$. solani choisie en fonction du problème pathologique posé: des fioles de Roux reçoivent $100 \mathrm{~g}$ de graines d'orge et $50 \mathrm{ml}$ d' $\mathrm{H}_{2} \mathrm{O}$; elles sont stérilisées à $110^{\circ} \mathrm{C}$ pendant $1 \mathrm{~h}$ à 2 jours d'intervalle. On rajoute, par fiole, $50 \mathrm{ml}$ d'une solution contenant pour 1 litre : chlortétracycline : $0,5 \mathrm{~g}$, sulfate de streptomycine : $0,5 \mathrm{~g}$, oxgall : $1 \mathrm{~g}$; le pH est ajusté à 4,5 avec $\mathrm{H}_{3} \mathrm{PO}_{4}$. Les fioles sont à nouveau stérilisées et, trois ou quatre jours après, elles sont inoculées au moyen de 10 explants de $1 \times 1$ $\mathrm{cm}$ répartis sur la surface des graines et prélevés à partir de cultures de $R$. solani ayant poussé 10 à 12 jours à $25^{\circ} \mathrm{C}$ sur du milieu PDA. Au bout de 15 jours de croissance à cette température, l'orge colonisée est séchée, broyée et calibrée sur un tamis de $3,15 \mathrm{~mm}$ de maille dans des conditions aseptiques.

L'inoculum est mélangé, à raison de $0,5 \%$ en poids, avec la quantité de sol nécessaire pour la mesure. Ce mélange, humidifié à $60 \%$ de sa capacité de rétention en eau, est mis en incubation à $25^{\circ} \mathrm{C}$.

Au moyen de l'essai biologique utilisant la plante sensible (cf.: I. 3) correspondant à l'identité de la souche de $R$. solani (cf.: I. 2. b), on mesure le PI (UPI $50 / \mathrm{g}$ ) du mélange à T0 (moment du mélange) puis à T30, T60, T90, T120 jours après. Les courbes de réceptivité sont présentées sur un graphique où l'on porte en abscisse le temps en jours et en ordonnée le logarithme des UPI 50/g. A partir du niveau de $\mathrm{Pl}$, obtenu à T0, les courbes matérialisant les mesures faites aux 4 dernières dates peuvent s'élever, s'abaisser ou rester proches de l'horizontale. Le sol est jugé comme réceptif (ou sensible), non réceptif (ou résistant), intermédiaire (ou tolérant).

Par rapport à la mesure à To, une diminution ou une augmentation des valeurs de $\mathrm{PI}$, supérieures à 1 ou 2 unités de l'échelle logarithmique (log UPI 50/g), signifie que l'influence du sol en faveur ou en défaveur de l'expression du pathogène a une probabilité d'être constatée en conditions naturelles.

\section{3) Capacité d'accueil du sol}

Elle est jugée en mesurant, pendant un temps donné, les variations de la CS pour l'inoculum d'une souche de $R$. solani qui a été introduite dans le sol.

La démarche technique est la même que pour la réceptivité (cf. II. 2) : les valeurs de CS obtenues à différents temps (T30, T60, T90, T120), comparées à la valeur initiale mesurée à T0, peuvent croître, décroître ou demeurer stables : la Capacité d'Accueil est positive, négative ou intermédiaire.

Si on démontre, pour une situation pathologique donnée, une corrélation entre la réceptivité et la capacité d'accueil, les études (épidémiologie, efficacité des moyens de lutte, etc...) peuvent être poursuivies de façon plus rapide (6 jours contre 13), plus simple (en boîte de Petri) et reproductible.

\section{4) Mesure de la réceptivité et de la capacité d'accueil à R. solani pour 3 sols naturels}

Le but de cette expérimentation a été d'étudier l'influence exercée par 3 sols naturels sur le comporte- 
ment parasitaire et saprophytique d'un inoculum de $R$. solani qui y a été introduit.

Trois sols naturels : A (terre maraîchère sablo-argileuse), B (terre betteravière argilo-limoneuse) et $C$ (terre d'alluvion à limon battant) ont été enrichis, à raison de $0,5 \%$ en poids, avec l'inoculum d'une souche de $R$. solani (AG4) très agressive sur Vigna radiata (cf. I. 3). Chaque sol a été divisé en 3 lots : 2 lots reçoivent respectivement un traitement fongicide (Basitac - PM à $75 \%$ de mépronil - Schering) à 0,5 et 1 $\mathrm{g}$ de $\mathrm{MA} / \mathrm{m}^{2}$, le troisième non traité sert de témoin. $\mathrm{Ce}$ traitement fongicide est destiné à induire des modifications dans le milieu sol afin de préciser les relations entre parasitisme et saprophytisme. La réceptivité (cf. II. 2) et la capacité d'accueil (cf. II. 3) de chaque combinaison ont été appréciées sur une période de 90 jours (Fig. 4).

Les résultats obtenus pour les témoins montrent qu'à T0, il y a concordance entre parasitisme et saprophytisme; ceci est logique puisque les sols n'ont pas eu le temps d'exercer leur influence sur l'inoculum introduit. En revanche, l'évolution au cours du temps prouve que chaque sol a une influence particulière dans la réceptivité et la capacité d'accueil : concordance pour le sol $A$, influence plus marquée sur la réceptivité dans le sol $B$, divergence pour le sol $C$ où une résistance progressive se manifeste pour la réceptivité tandis que la capacité d'accueil évolue peu. Pour ce dernier sol, il est intéressant de savoir que $R$. solani y occasionne de graves attaques sur les cultures de salades en plein air : il s'agit, à notre sens, d"un exemple où la culture d'une plante sensible modifie l'influence propre exercée par le sol sur le comportement parasitaire, en levant une fongistase naturelle.

Les modifications induites par le traitement fongicide sont assez fidèlement reproduites pour chaque sol, à la fois pour le parasitisme et le saprophytisme puisque la forme des courbes obtenues dans les deux cas est sensiblement comparable. Les variations marquées de ces courbes s'expliquent par le comportement de l'inoculum artificiel utilisé et le mode d'action du fongicide. Durant la préparation de l'inoculum (cf. II. 2), on observe des poussées successives de croissance du champignon sur l'orge; l'inoculum final est composé d'un mélange de deux parties rentrant en activité de façon différente: l'une d'elles assure la colonisation rapide du sol dès la mise en place (TO), l'autre, composée de formes de résistance, est à l'origine d'une seconde colonisation plus tardive (T60). La diminution des valeurs de $\mathrm{PI}$ et de CS à T30 et T90 correspond à l'effet produit par le Basitac qui est un fongicide de contact très efficace sur les myceliums en phase de croissance active. On remarque, par ailleurs, que l'efficacité du fongicide augmente avec la dose d'application dans tous les cas.

\section{5) Epidémiologie}

La fixation expérimentale dans l'écosystème (cf. II. 1) des paramètres : inoculum, plante, climat, permet d'apprécier l'influence exercée par le sol sur le parasite. Mais il s'agit d'une phase ponctuelle dans l'évolution biologique du sol : les variations naturelles du climat, les espèces végétales cultivées entraînent des modifications du milieu sol qui, par action sur l'inoculum qu'il contient, vont moduler l'intensité des attaques sur la plante. II est possible d'approcher la compréhension de l'épidémiologie des maladies dues à $R$. solani. Les incubations des mélanges : sol + inoculum, sont sou- mises à l'influence de la culture de différentes plantes ou à des variations des éléments du climat, d'abord de manière individuelle, puis en combinaison. On juge les modifications induites par la variation de la réceptivité des différentes combinaisons. Les études épidémiologiques par ce moyen sont engagées dans notre laboratoire.

\section{Lutte}

L'utilisation des techniques d'appréciation de la réceptivité et de la Capacité d'Accueil, en permettant de faire la part de l'influence du sol, sert à juger plus précisément l'efficacité de moyens de lutte.

Nous avons axé le programme de lutte contre $R$. solani dans deux directions : lutte classique par utilisation de fongicides, lutte biologique au moyen du champignon antagoniste Trichoderma. Les essais sont réalisés dans des sols naturels "expérimentaux", en ce sens que l'on a mesuré au préalable leur influence sur le parasitisme et le saprophytisme du parasite.

\section{1) Utilisation des fongicides}

L'application au champ d'une matière fongicide est précédée d'une étude d'efficacité au laboratoire en utilisant les essais biologiques (cf. II. 1): différentes doses du fongicide sont amenées, en mélange dans la masse ou par arrosage, dans la couche de sol infesté par $R$. solani au moment de l'inoculation des plantules. Il est ainsi possible de connaître rapidement la dose et le mode d'apport assurant la meilleure protection.

Une variante intéressante de cette technique consiste à prélever le sol préalablement traité au champ dans les conditions de la pratique et, après enrichissement avec l'inoculum d'une souche du parasite adaptée au problème posé, à comparer les intensités de maladies notées dans les échantillons traités avec celle d'un témoin du même sol non traité. On peut ainsi connaître non seulement l'efficacité du fongicide mais également la validité de la méthode d'application.

\section{Essai d'efficacité de fongicides}

Dans la région de Lille (59), les cultures de chicorées frisées sont affectées par une grave pourriture du cœur. Les attaques ont été attribuées à $R$. solani et les souches isolées à la fois sur la plante et dans le sol ont pu être rattachées au groupe d'anastomose 1 ( $c f$. I. 2. b).

Un sol, provenant d'une exploitation où la pourriture sévit particulièrement, a été enrichi, à raison de $0,5 \%$ en poids, avec l'inoculum d'une souche isolée sur la plante et très agressive sur Vigna radiata.

Trois fongicides ont été utilisés (cf. III. 1) : l'efficacité de matières actives expérimentales : MoncerenBayer (PM à 12,5\% de pencycuron), Basitac-Schering (PM à $75 \%$ de mépronil) a été comparée à celle de Rovral-Rhône Poulenc (PM à 50\% d'iprodione), fongicide de référence. Les résultats obtenus sont présentés dans la figure 5.

Pour Monceren et Basitac, le traitement de sol en mélange dans la masse permet d'obtenir des indices de maladies beaucoup plus faibles que le traitement par arrosage. Par ailleurs, ces 2 fongicides ont un effet 

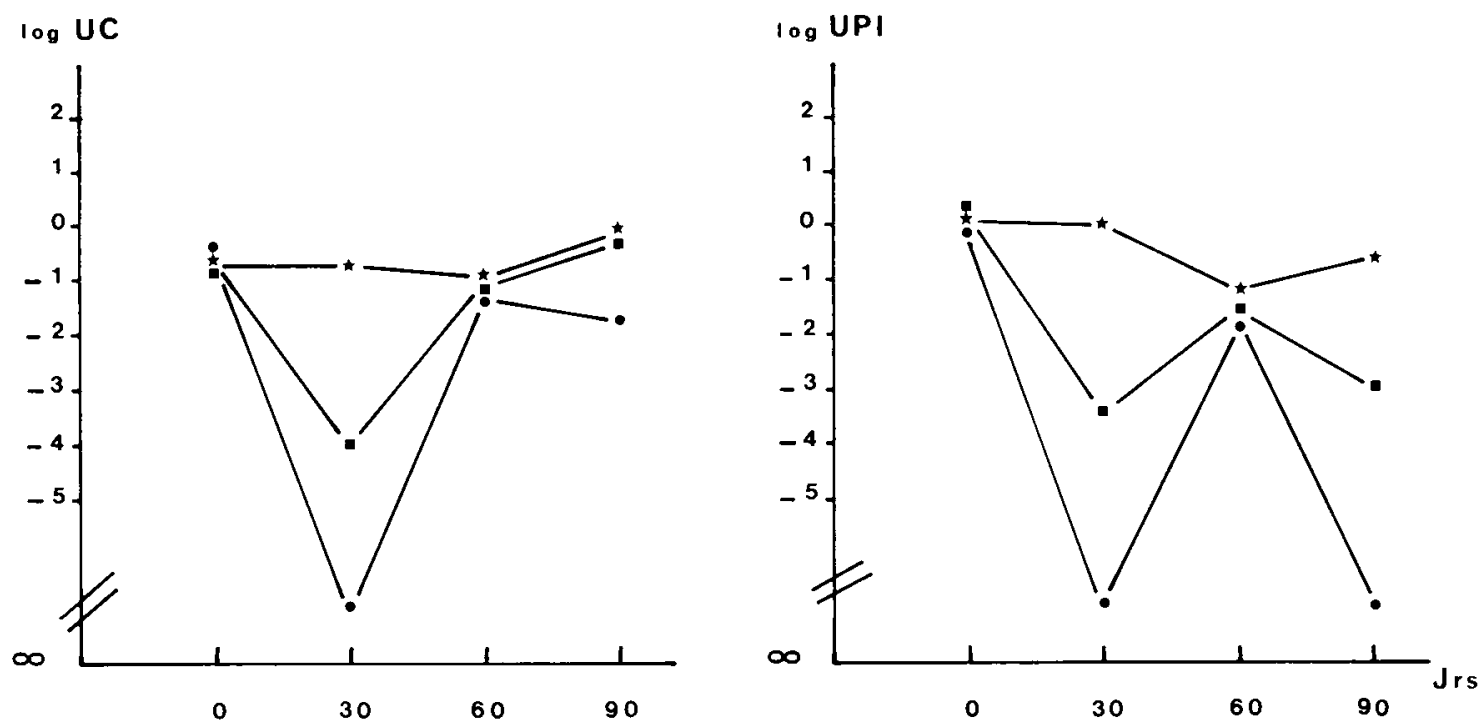

A
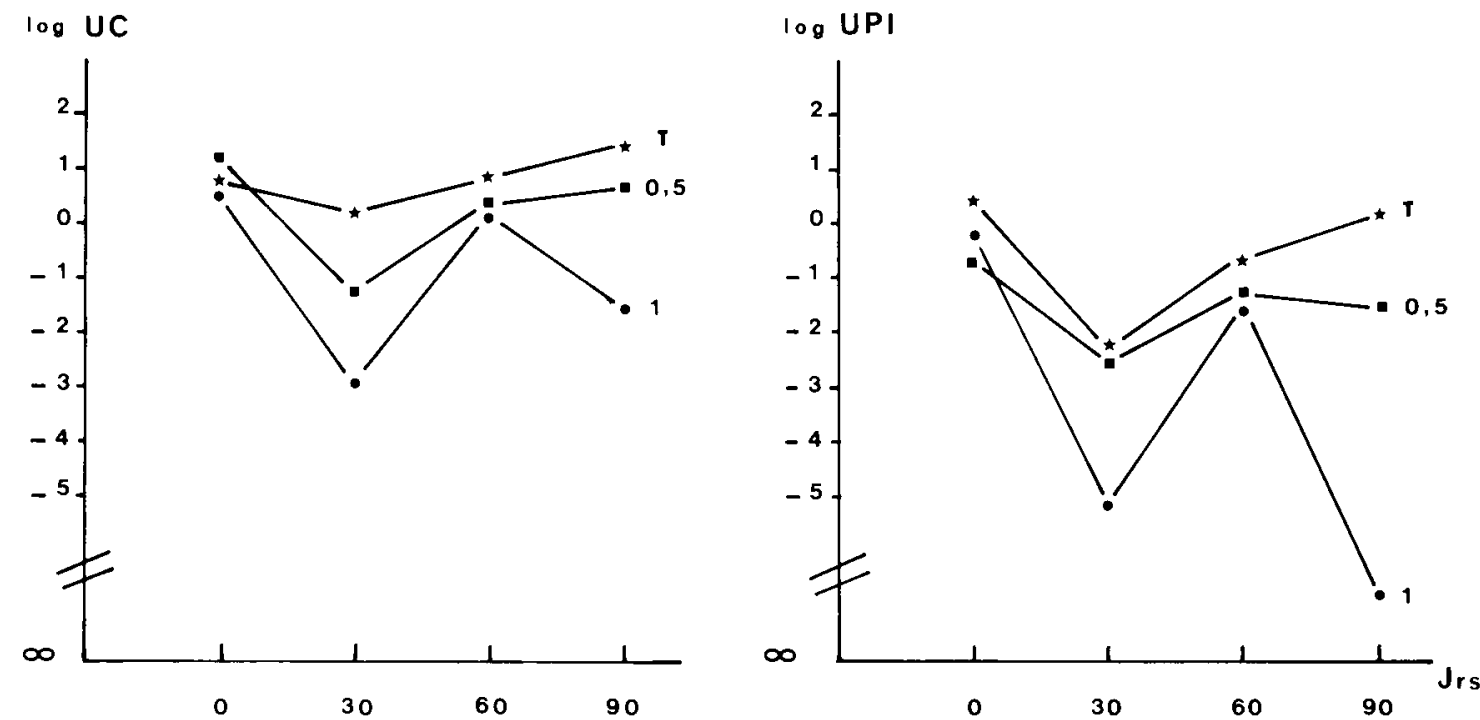

B
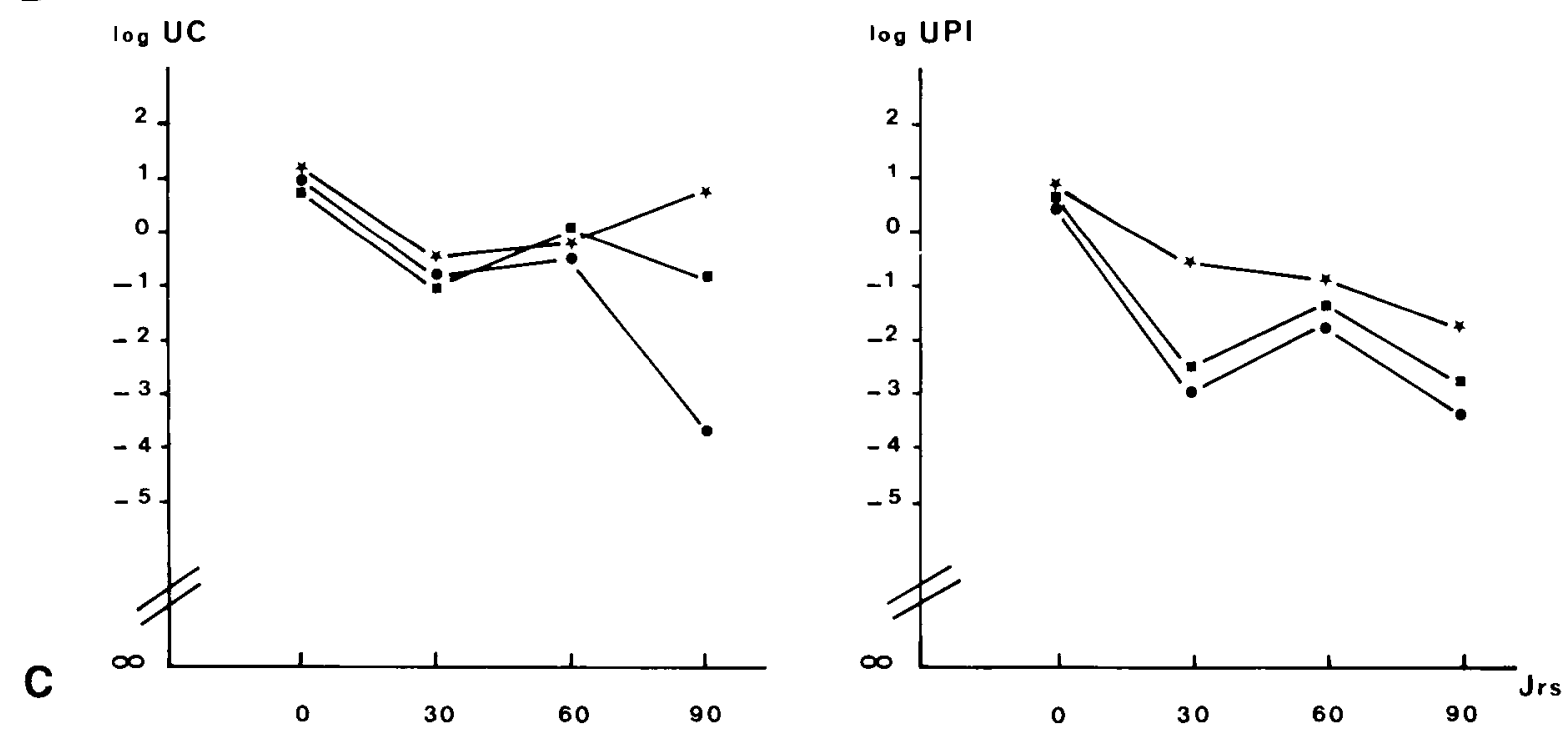

Fig.4. Réceptivité et capacité d'accueil pour $R$. solani, de 3 sols naturels traités par un fongicide. $A=$ terre maraîchère sablo-argileuse, $\mathrm{B}=$ terre betteravière argilo-limoneuse, $\mathrm{C}=$ terre maraîchère limon à battant. L'axe des abscisses porte le temps exprimé en jours. L'axe des ordonnées, dans les graphiques situés à gauche, porte le logarithme des unités de colonisation $50 / \mathrm{g}$ (log UC), pour les graphiques situés à droite, le logarithme des unités de potentiel infectieux 50/g (log UPI). Pour les différentes combinaisons, la réceptivité est matérialisée dans la partie droite et la capacité d'accueil dans la partie gauche. Témoin non traité $\star T$, sol traité par Basitac à $0,5 \mathrm{~g} \mathrm{MA} / \mathrm{m}^{2} \bullet$, sol traité par Basitac à $1 \mathrm{~g} \mathrm{MA} / 2^{2} \bullet$. 
dépressif sur les attaques de $R$. solani nettement plus élevé que Rovral.

\section{2) Lutte biologique}

L'efficacité du contrôle biologique de la maladie au moyen de Trichoderma spp. dépend du choix des souches du champignon qui doivent pouvoir s'établir dans le sol et y exercer leurs capacités antagonistes.

Dans un premier temps, la mise au point de techniques autorisant l'étude du pouvoir antagoniste des souches in vitro (Camporota, 1985) et leur aptitude au saprophytisme dans les sols (Camporota, 1983; Davet et Camporota, 1986) a permis d'opérer un choix parmi les souches de nos mycothèques.

La deuxième phase, en cours d'achèvement, a eu pour but d'apprécier, en chambre climatisée puis en serres, l'antagonisme des souches de Trichoderma spp. vis-à-vis de $R$. solani. Nous présentons, à titre d'exemple, les résultats obtenus pour 2 souches de Trichoderma spp. : $\mathrm{HH}_{3}$ et $\mathrm{T}_{43}$, au cours de l'expérimentation en chambre climatisée :

- $\mathrm{HH}_{3}$, agressive sur Sclerotinia sclerotiorum (Artigues et Davet, 1984);

- $T_{43}$, présentant un fort mycoparasitisme sur $R$. solani (Camporota, 1985) (Tableau I).

Pour ces souches, un inoculum a été produit sur de la pulpe de betterave en conditions non aseptiques en utilisant le milieu sélectif de Davet (1979). Un sol naturel, enrichi par l'inoculum d'une souche agressive de $R$. solani (AG4), a été traité au moyen de suspensians de spores des souches antagonistes.

Pour chaque combinaison, la quantité de sol nécessaire a été mise en incubation pendant 2 mois afin de permettre l'installation du phénomène biologique, et l'évolution du comportement parasitaire de $R$. solani a été mesurée au moyen de l'essai utilisant Vigna radiata (cf. I. 3). Les mesures ont été faites à 5 dates: à T0 (au moment de la mise en place) puis à T8, T15, T30, T60 jours après. (Tableau II).

A T60, la souche T43 a permis d'obtenir des IM significativement différents $\left(^{*}\right)$ de ceux mesurés pour le témoin tandis que ceux correspondant à $\mathrm{HH} 3$ sont à la limite de signification.

Malgré l'utilisation de quantités de spores/g de sol relativement élevées, les résultats pour T43 sont prometteurs car l'essai biologique, en assurant des conditions optimales pour l'expression pathogène de $R$. solani, conduit à une pression parasitaire rarement présente en conditions naturelles.

\section{Discussion}

II est évident que des moyens de lutte applicables dans la pratique sont obtenus au terme de

Tableau I. Nombre de spores de Trichoderma spp. pour $1 \mathrm{~g}$ de sol infecté par $R$. solani. Plan de l'expérimentation.

\begin{tabular}{|c|c|c|c|c|}
\hline $10^{7}$ & $10^{8}$ & $10^{7}$ & $10^{8}$ & 0 \\
\hline \multirow[t]{2}{*}{+} & + & + & + & + \\
\hline & & & & Témoin \\
\hline
\end{tabular}
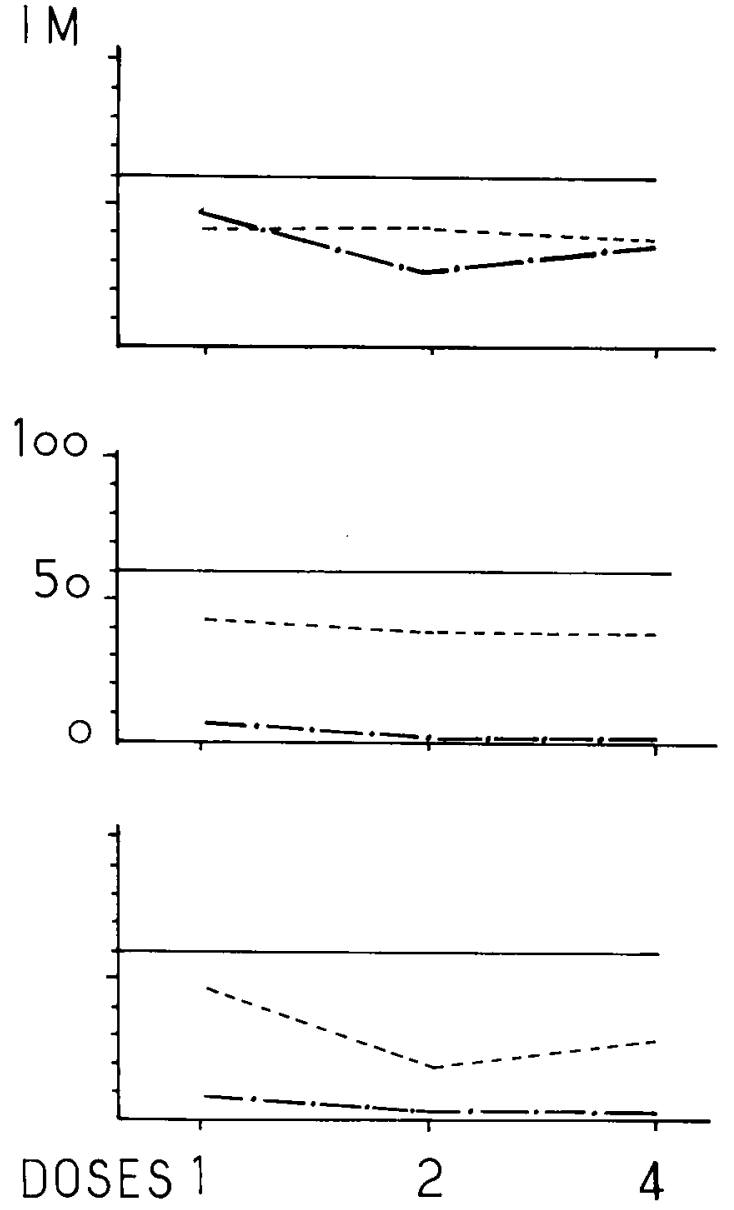

Fig. 5. Efficacité, comparée au laboratoire, de 3 fongicides destinés à contrôler les attaques de $R$. solani sur Vigna radiata: A (Rovral); B (Monceren); C (Basitac). L'axe des abscisses porte les doses de matière active $\left(g \mathrm{MA} / \mathrm{m}^{2}\right)$ celui des ordonnées, les indices de maladie en \% (IM). (- $\longrightarrow$ ): témoin non traité, (- - - - ) : traitement du sol par arrosage, $(-\cdot-\cdot-)$ : mélange du fongicide dans la masse du sol.

leur expérimentation au champ. Mais la mise en œuvre de cette expérimentation présente un certain nombre d'inconvénients : (i) choix de la parcelle expérimentale qui doit être de taille suffisante pour l'étude statistique et bien pourvue en inoculum agressif bien réparti, (ii) les conditions climatiques peuvent conduire à une absence de maladie quand elles sont défavorables, (iii) la durée de la culture ne permet pas de répéter l'expérimentation dans les mêmes conditions d'environnement pour la majorité des cas.

II est illusoire d'intervenir sur les 2 derniers points, en revanche, l'inoculation artificielle permet de résoudre en partie le premier, mais de façon onéreuse et même dangereuse (dissémination de la maladie).

Tous ces inconvénients conduisent à la conclusion qu'il est important de posséder des éléments de compréhension et de décision permettant la mise en place d'une expérimentation ayant des chances d'aboutir à des résultats justifiant l'investissement engagé. 
Tableau II. Indices de maladie (IM) obtenus à chaque date d'analyse pour l'ensemble des combinaisons. Les valeurs d'IM suivies de (") sont significativement différentes au seuil de confiance de $5 \%$.

\section{Dates d'analyse}

\begin{tabular}{|c|c|c|c|c|c|c|}
\hline \multicolumn{3}{|c|}{ Nbre spores/g } & \multirow[b]{2}{*}{ T8 } & \multirow[b]{2}{*}{$T 15$} & \multirow[b]{2}{*}{ T30 } & \multirow[b]{2}{*}{$T 60$} \\
\hline & de sol & TO & & & & \\
\hline \multirow{2}{*}{$\mathrm{HH3}$} & $10^{7}$ & 91 & 78 & 68 & 85 & 62 \\
\hline & $10^{8}$ & 89 & 73 & 73 & 69 & 70 \\
\hline \multirow{2}{*}{ T43 } & $10^{7}$ & 96 & 80 & 61 & 74 & $40^{*}$ \\
\hline & $10^{8}$ & 92 & 86 & 58 & 61 & $31^{*}$ \\
\hline Témoin & 0 & 97 & 88 & 93 & 89 & 78 \\
\hline
\end{tabular}

L'ensemble des techniques présentées permettent de répondre à cette préoccupation. II est possible d'expérimenter, tout au long de l'année et de comparer l'influence exercée par les sols sur une maladie reproduite de façon régulière et standardisée.

Certes, ces moyens d'étude sont artificiels et on peut penser que ne reflétant pas la réalité biologique du champ, il sera difficile d'exploiter les résultats obtenus pour l'expérimentation pratique.

Les travaux menés dans notre laboratoire pour résoudre deux problèmes posés par la profession (pourriture basale de la laitue, pourriture du cœur de la chicorée) ont montré le contraire : l'application du plan d'étude que nous avons exposé a permis de déterminer $R$. solani AG1 comme agent causal et d'établir la relation entre les souches isolées sur la plante et dans le sol, puis de choisir des parcelles naturellement infestées pour l'expérimentation au champ, enfin de pouvoir procéder au laboratoire à l'étude d'efficacité des molécules fongicides avec des souches de $R$. solani spécifiques et très agressives. La transposition au champ de ces résultats a abouti à un contrôle efficace de la maladie tant pour la laitue (Camporota et al., 1986; Camporota et al., 1988) que pour la chicorée frisée (Programme INRA - PV de Lille, en cours).

L'utilisation de ces techniques trouve en outre son intérêt dans le cas de la lutte biologique au moyen deTrichoderma $s p$ : la mise en confrontation de micro-organismes aux potentialités biologiques multiples conduit à l'obligation de pouvoir juger des interrelations entre le sol, le parasite, l'antagoniste, sous l'influence des autres facteurs du milieu (plante, climat, traitements agrono- miques). Les connaissances que nous obtenons au cours de nos travaux, sont d'une aide essentielle pour pouvoir utiliser dans la pratique ce moyen de lutte très intéressant.

Pour conclure, nous dirons qu'il est nécessaire d'aborder d'autres problèmes pathologiques dûs à $R$. solani de la même manière afin de pouvoir étalonner plus précisément et de perfectionner encore ces moyens d'étude que nous avons élaborés.

\section{Remerciements}

L'auteur tient à remercier sa femme, Reine Camporota pour sa collaboration bénévole au cours des recherches.

\section{Références}

Aouchiche H. (1982) Recherche d'une méthode de mesure du potentiel infectieux de sols infestés par Rhizoctonia solani, agent du rhizoctone brun de la pormme de terre. DEA, ENSSAA Rennes.

Artigues M. \& Davet P. (1984) Comparaison des aptitudes parasitaires de clones de Trichoderma vis-à-vis de quelques champignons à sclérotes. Soil Biol. Biochem. 16, 413-417

Camporota P. (1981) Mesure de la colonisation saprophytique en compétition de Rhizoctonia solani Kühn dans les sols et substrats. Agronomie 1, 6, 531-517

Camporota P. (1982) Recherches sur l'écologie des champignons parasites du sol. XVII. Mesure du potentiel infectieux de sols et substrats infestés par Rhizoctonia solani Kühn, agent de fontes de semis. Agronomie 2, 5, 437-442

Camporota P. (1983) Evolution du comportement saprophytique de souches de Trichoderma dans les sols. Technique d'étude. Agronomie 3, 6, 607-609

Camporota P. (1985) Antagonisme in vitro de Trichoderma spp. vis-à-vis de Rhizoctonia solani Kühn. Agronomie 5, 7, 613-620

Camporota P., Lucas P. \& Aouchiche (1984) Variabilité du genre Rhizoctonia. In : Variation et variabilité des agents phytopathogènes Avignon 10-11 mai 1984, Coll. de I'INRA, 26, pp. 187-191

Camporota P., Baudrand M. \& Taussig C. (1986) Pourriture basale de la laitue provoquée par Rhizoctonia solani : essais de traitements au champ. PHM 271, 3338

Camporota P., Baudrand M. \& Taussig C. (1988) Nouvelles molécules actives contre Rhizoctonia solani agent de la pourriture basale de la laitue. Deux années d'expérimentation au champ. PHM 293, 44-50

Davet P. \& Camporota P. (1986) Etude comparative de quelques méthodes d'estimation de l'aptitude à la compétition saprohytique dans le sol des Trichoderma. Agronomie 6, 6, 575-581

Davet P. (1979). Technique pour l'analyse des populations de Trichoderma et de Gliocladium virens dans le sol. Ann. Phytopathol. 11, 529-533

Geypens M. (1974) Inoculum potential of soil borne plant pathogenic fungi : problems encountered in analysis and significance in epidemiology. Agro - Ecosystems 1, 177-192 
Papavizas G.C. (1970) Colonization and growth of Rhizoctonia solani in soil. In : Rhizoctonia solani : Biology and Pathology. Univ. California Press, Berkeley, Los Angeles and London, pp. 108-122

Parmeter J.R., Sherwood R.T. \& Platt W.D. (1969) Anastomosis grouping among isolates of Thanatephorus cucumeris. Phytopathology 59, 1270-1278

Parmeter J.R \& Whitney H.R. (1970) Taxonomy and nomenclature of the imperfect stage. In : Rhizoctonia solani : Biology and Pathology. Univ. California PressBerkeley, Los Angeles and London, pp. 7-19

Ricci P. (1972) Moyens d'étude de l'inoculum du Phytophthora nicotianae f. sp. parasitica (Dastur) Waterh., parasite de l'œillet dans le sol. Ann. Phytopathol. 4, 3, 257-276

Sneh B., Katan J., Henis Y. \& Wahl I. (1966) Methods for evaluating inoculum density of Rhizoctonia in naturally infested soil. Phytopathology 56, 74-78 